\title{
Resonating Valence Bond Mechanism of Impurity Band Superconductivity in Diamond
}

\author{
G. Baskaran ${ }^{1,2}$ \\ ${ }^{1}$ Institute of Mathematical Sciences \\ Chennai 600 113, India \\ ${ }^{2}$ IFCAM \& Institute for Materials Research \\ Tohoku University \\ Sendai 980-8577, Japan
}

\begin{abstract}
Superconductivity in an uncompensated boron doped diamond, a very recent observation, is strikingly close to an earlier observation of Anderson-Mott insulator to metal transition, prompting us to suggest an electron correlation driven superconductivity in an impurity band. Random coulomb potential remove a three fold orbital degeneracy of boron acceptor states, resulting in an effective single, narrow, tight binding and half filled band of holes. Singlet coupling between spins of neighboring neutral acceptors $B^{0}-B^{0}$ is the seed of pairing. Across the insulator to metal transition, a small and equal fraction of charged $B^{+}$and $B^{-}$states (free carriers) get spontaneously generated and delocalize. Thereupon neutral singlets resonate and get charged resulting in a resonating valence bond (RVB) superconducting state.
\end{abstract}

PACS numbers:

Diamond has been reported [1] to become a superconductor upon high boron doping with a $T_{c} \approx 4 K$. This remarkable discovery of superconductivity in an uncompensated p-type semiconductor has possible implications for basic science and technology. As an example, shallow dopant states in $\mathrm{Si}$ and Ge play a key role in modern solid state electronics; from physics point of view they offer a testing ground for various ideas such as Anderson and Mott localization and their interplay. Boron doped diamond [2], being a relatively deep level acceptor with a hole binding energy of $\approx 0.37 \mathrm{eV}$ provides a new energy scale, new possibilities [3] and a rich physics. Further investigation of this system should be rewarding.

The present problem of electronic phases of boron doped diamond, containing competing coulomb interaction, randomness and coupling to phonon, is like Si:P, quite a complex and challenging many body problem 4 . However, using a body of insights one has gained in the last couple of decades from theory and experiments one can suggest physically plausible mechanism and insights, which could be guiding hypothesis to understand the observed superconductivity in boron doped diamond (diamond:B). It is in this spirit, the present letter suggests a minimal model and mechanism for superconductivity. The dopant density seems 7 ] to be just above the critical density of the Anderson-Mott transition making electron correlation important. We suggest a resonating valence bond (RVB) mechanism of superconductivity 5, 6, 9] in boron impurity band system. Diamond, a broad band insulator, where electron correlations are not important, should be viewed as offering an appropriate vacuum to boron (atom with an odd electron number) subsystem, where electron correlation driven Anderson-Mott insulator to superconductor transition and other rich correlation physics could take place(figure 1).

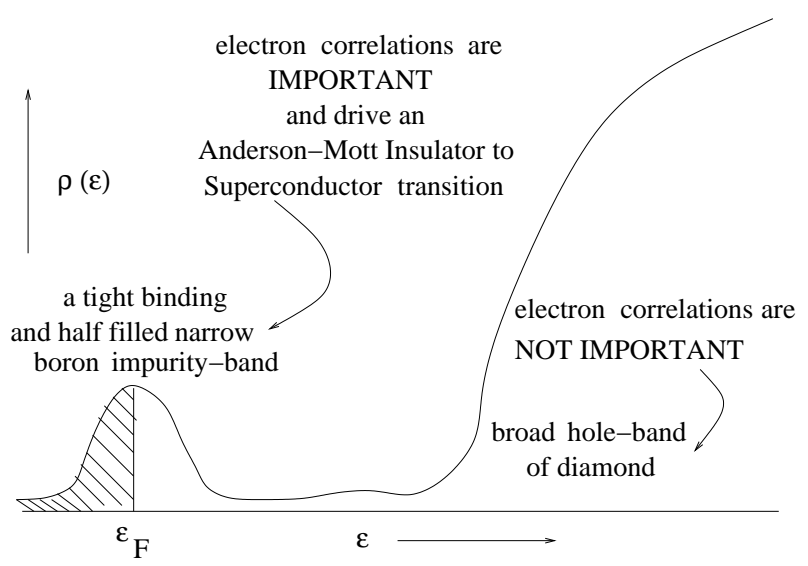

FIG. 1: Hole density of states (schematic) in boron doped diamond, an uncompensated p-type semiconductor. Holes of acceptors form a strongly correlated and effective single impurity band at half filling. Anderson-Mott insulator to superconductor transition is suggested to take place in the impurity band as we increase boron density (figure 2).

The observed superconductivity [1] in diamond:B is of type-II with a $T_{c} \approx 4 \mathrm{~K}, H_{c 2} \geq 3.5 \mathrm{~T}$ and coherence length $\xi_{\mathrm{GL}} \sim 100 \mathrm{Au}$. The dopant boron concentration is around $4.5 \times 10^{21} / \mathrm{cm}^{3}$.

Transport properties of diamond:B has been studied by various authors and the issue of metal insulator transition in the impurity band discussed $7,[8]$. The only report to my knowledge, which makes a low temperature measurement of the critical boron concentration by a scaling analysis is by Tshepe et al. [7]. They find a critical doping concentration $n_{c} \approx 4 \times 10^{21} / \mathrm{cm}^{3}$, strikingly close to the boron concentration $\sim 4.5 \times 10^{21} / \mathrm{cm}^{3}$ used in the new superconductor [1]. It is this closeness which prompted us to examine the role of electron corre- 
lation and suggest a Anderson-Mott insulator to RVB superconductivity transition, similar to the one advocated recently [9, 10] in the context of (non-random) Mott insulator to superconductor transition. Importance of strong correlation is further enforced by a nearly temperature independent and a very large value of normal state resistivity of the (polycrystalline) superconducting sample 1], $\rho \sim 9 \mathrm{~m} \Omega \mathrm{cm}$, which gives a mean free path $\ell_{0} \sim 0.1 \mathrm{Au}$, much less than the average separation between neighboring acceptor sites. Resistivity in single crystal or low frequency conductivity measurements in polycrystalline sample will be welcome to substantiate this point.

Diamond, an excellent insulator has a very broad band and a wide gap $\sim 5.6 \mathrm{eV}$. The top of the valence band is three fold degenerate with effective masses, $2.12 m_{e}, 0.7 m_{e}$ and $1.06 m_{e}$. Boron, which has one less electron compared to a carbon, becomes an acceptor, when it substitutes a carbon atom in diamond [2]. The acceptor states are threefold degenerate with a hole binding energy of $E_{B} \approx 0.37 \mathrm{eV}$. Spin-orbit coupling partly removes this degeneracy by a marginal $6 \mathrm{meV}$.

The relative dielectric constant of diamond is $\epsilon_{0} \sim 5.6$. Effective mass theory estimate of acceptor states gives a hydrogenic impurity state with an effective Bohr radius of $a_{B}^{*} \equiv \frac{e^{2}}{2 \epsilon_{0} E_{B}} \sim 3 A u$, of the 'envelope function'. When this is used in conjunction with Mott's criterion of Mott insulator metal transition, $n_{c} a^{3} \approx 0.25$, one obtains 11 a critical doping, $n_{c} \approx 8.0 \times 10^{21} / \mathrm{cm}^{3}$. Experimentally observed critical concentration of reference [7] is of the same order as this rough estimate.

Keeping the above in mind, we build a simple tight binding model for the impurity band. The three fold degenerate acceptor wave functions (transforming as $\left.t_{2 g}\right)$ have different spatial density distribution $\mid \psi_{\alpha}(\mathbf{r}-$ $\left.\mathbf{R}_{i}\right)\left.\right|^{2}$,for $\alpha=1,2$ and 3. Here $\mathbf{R}_{i}$ is the site of a boron atom. Hence the total electrostatic potential a hole in an acceptor state feels from neighboring dopants depends on the orbital it occupies. In other words, the three fold degeneracy of an acceptor state is in general lifted by the randomness. Simple estimate shows that the amount of lifting, for the boron doping of interest, is large compared to the impurity band width $\sim 0.2 \mathrm{eV}$. For low energy physics of interested to us, only the lowest energy acceptor states are important. This is also consistent with general experience in $\mathrm{Si}: \mathrm{P}$, where the degeneracy of donor states has no substantial role and a non-degenerate donor state theory seem to work well [12].

Once we identify a lowest energy acceptor state for each boron, a one band tight binding model Hamiltonian follows:

$$
\begin{aligned}
H & \approx \sum_{i} \epsilon_{i} c_{i \sigma}^{\dagger} c_{i \sigma}-\sum t_{i j} c_{i \sigma}^{\dagger} c_{j \sigma}+h . c . \\
& +\sum_{i} U_{i}\left(1-n_{i}\right)^{2}+\frac{1}{2} \sum W_{i j}\left(1-n_{i}\right)\left(1-n_{j}\right)(1)
\end{aligned}
$$

Here $c_{i \sigma}^{\dagger}$ is the hole creation operators at the lowest energy acceptor state of boron at site $\mathrm{i}$ with an energy $\epsilon_{i}$ and $t_{i j}$ are the hopping matrix elements; $n_{i} \equiv n_{i \uparrow}+n_{i \downarrow}$. The parameter $U_{i}$ is the Hubbard repulsion term of an acceptor state centered at site $\mathrm{i}$ and $W_{i j} \approx \frac{e^{2}}{\epsilon_{0} R_{i j}}$ is the diagonal coulomb matrix element between two acceptor states separated by a distance $R_{i j}$. The total number of holes is the same as the number of boron atoms; we have a half filled band of interacting holes.

On the insulating side of the above Hamiltonian, the low energy sector is the spin sector, which can be seen clearly in the limit $U>>t$ as a random antiferromagnetic Heisenberg Hamiltonian with superexchange interaction $J_{i j}$ :

$$
H_{s} \approx \sum_{i j} J_{i j}\left(\mathbf{S}_{i} \cdot \mathbf{S}_{j}-\frac{1}{4}\right)
$$

This is well established in the context of Si:P for example leading to notions of hierarchy of singlet spin coupling, valence bond localization, valence bond glass etc., both experimentally and theoretically $[13]$. We do not think diamond:B is fundamentally different from $\mathrm{Si}: \mathrm{P}$ in the Mott insulating side.

The issue for us is the conducting side close to Mott transition point. In the present paper, based on our recent study, we offer a new insight. Conventionally the conducting side is thought of as a disordered fermi liquid and with regions of certain local moment character 14. We have recently suggested $[9]$ that a corresponding conducting state close to the Mott transition point in the non-random case is well thought of as a 'self doped Mott insulator', a projected metal. In a self doped Mott insulator a small and equal density of free positive $\left(B^{+}\right)$ and negative $\left(B^{-}\right)$carriers are self consistently generated across the Mott transition, out of the neutral states $B^{0}$, in the process of minimizing the free energy, particularly the long range coulomb interaction part. Further, antiferromagnetic superexchange, that is characteristic of a Mott insulator survives in the conducting state as well, the same way superexchange survives in the $\mathrm{CuO}$ planes of cuprates in the presence of doped holes. That $i s$, in the above conducting state, in addition to 'virtual' double occupancy and empty sites, which are responsible for generation of superexchange, a small and equal density of 'real' and delocalized double occupancy and empty sites are maintained. Our work $[9]$ unified RVB mechanism of superconductivity in hole doped cuprates with that in organic superconductors such as 2D ET salts and also predicted new systems.

An effective Hamiltonian of the above conducting state is the 2 species random t-J Hamiltonian that is obtained [9] by a superexchange perturbation theory for the present situation of half filled band of disordered in- 
teracting electron Hamiltonian (equation 1)

$$
\begin{aligned}
H_{t J}= & -\sum_{i j} t_{i j} P_{d} c_{i \sigma}^{\dagger} c_{j \sigma} P_{d}-\sum_{i j} t_{i j} P_{e} c_{i \sigma}^{\dagger} c_{j \sigma} P_{e}+\text { h.c. } \\
& -\sum_{i j} J_{i j}\left(\mathbf{S}_{i} \cdot \mathbf{S}_{j}-\frac{1}{4} n_{i} n_{j}\right)+\sum_{i} \epsilon_{i} c_{\sigma}^{\dagger} c_{\sigma}
\end{aligned}
$$

operating in a subspace that contains a fixed number $x N$ of doubly occupied $B^{-}$and $x N$ empty $B^{+}$states. Here $N$ is the total number of electrons, which is the same as the number of lattice sites. The projection operators $P_{d}$ and $P_{e}$ allow for the hopping of charged $B^{-}$and $B^{+}$states respectively, maintaining $(1-2 x) N$ singly occupied neutral states $B^{0}$ in a dynamical fashion. Notice that the long range coulomb interaction has disappeared in the above Hamiltonian because of screening in the metallic state; More importantly it has left its effect parametrically through the generation of a finite density $2 x$ of free carriers by the process of self doping.

Our 2 species random t-J model adapted to the self doped Mott insulator has a more transparent form in the slave boson representation $c_{i \sigma}^{\dagger} \equiv s_{i \sigma}^{\dagger} d_{i}+\sigma s_{i \bar{\sigma}} e_{i}^{\dagger}$. Here the charge-ons $d_{i}^{\dagger}$ 's and $e_{i}^{\dagger}$ 's are hard core bosons that create doubly occupied sites $\left(B^{-}\right.$of charge $\left.e^{-}\right)$and empty sites $\left(B^{+}\right.$of charge $\left.e^{+}\right)$respectively. The fermionic spinon operators $s_{i \sigma}^{\dagger}$ 's create singly occupied sites $\left(B^{0}\right.$, charge neutral) with a spin projection $\sigma$. The local constraint, $d_{i}^{\dagger} d_{i}+e_{i}^{\dagger} e_{i}+\sum_{\sigma} s_{i \sigma}^{\dagger} s_{i \sigma}=1$, keeps us in the right Hilbert space.

In the slave boson representation our t-J model takes a suggestive form:

$$
\begin{aligned}
H_{t J} & =-\sum_{i j} t_{i j}\left(e_{i}^{\dagger} e_{j} \sum_{\sigma} s_{i \sigma}^{\dagger} s_{j \sigma}-d_{i}^{\dagger} d_{j} \sum_{\sigma} s_{i \sigma}^{\dagger} s_{j \sigma}\right)+h . c . \\
& -\sum_{i j} J_{i j} b_{i j}^{\dagger} b_{i j}+\sum_{i} \epsilon_{i}\left(d_{i}^{\dagger} d_{i}-e_{i}^{\dagger} e_{i}\right)
\end{aligned}
$$

where $b_{i j}^{\dagger}=\frac{1}{\sqrt{2}}\left(s_{i \uparrow}^{\dagger} s_{j \downarrow}^{\dagger}-s_{i \downarrow}^{\dagger} s_{j \uparrow}^{\dagger}\right)$ is a spin singlet spinon pair creation operator at the bond $i j$.

At a formal level, a slave boson analysis is readily performed for our random interacting electron model. We suggest two different phases, a valence bond glass (VBG) and RVB superconducting phase, shown in figure 2 .

In the Anderson-Mott insulator region we have a VBG phase. This is brought out well [13] both in experiments and theory in the case of Si:P. This state contains only real 'neutral' $B^{0}$ states and no real $B^{+}$and $B^{-}$charged states. A good variational wave function to describe such a valence bond glass state is

$$
|V B G\rangle=\hat{P}\left(\sum_{i j} \phi_{\mathrm{VBG}}(i, j) b_{i j}^{\dagger}\right)^{N}|0\rangle,
$$

that describes condensation of neutral valence bonds in a specific valence bond pattern given by the pair function $\phi_{\mathrm{VBG}}(i, j)$. While one can formally write down a self

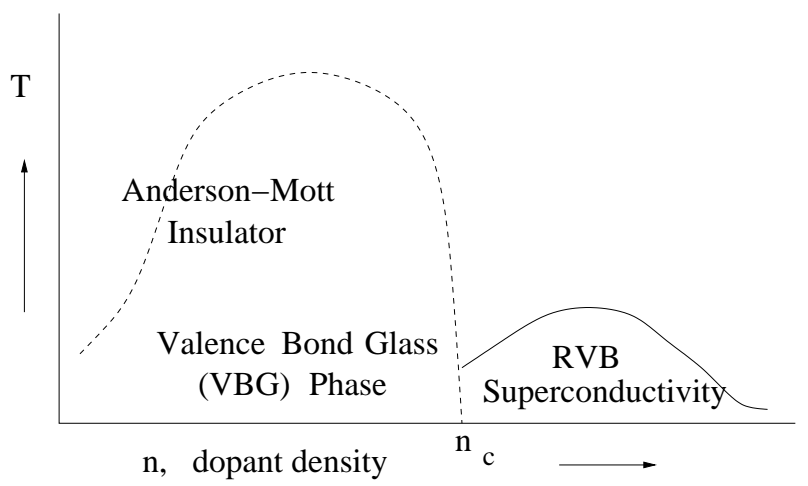

FIG. 2: Schematic Phase Diagram as a function of dopant density in Diamond:B, an uncompensated case.

consistent gap equation for the pair function $\phi_{\mathrm{VBG}}(i, j)$ of our random correlated spin-half system, one does not attempt to solve it but assumes that a solution exists.

In $\mathrm{Si} P$, the hierarchically organized valence bond couplings 13 in a VBG leads to a 'pseudo gap' that gives a magnetic susceptibility that vanishes as $\chi_{\text {spin }} \sim \frac{1}{T^{1-\alpha}}$ with $\alpha>0$. The spin contribution to specific heat also gets a similar power law correction.

As mentioned earlier, across the Mott transition a small and equal density of positive and negative carriers are spontaneously generated out of the insulating valence bond glass state. The delocalization of charged carriers causes charging and resonance of the frozen valence bonds resulting in an RVB superconducting state. This state is best described by a generalized RVB variational wave function, inferred from a slave boson mean field analysis:

$$
|R V B\rangle=\hat{P}\left(e_{\mu}^{\dagger}\right)^{x N}\left(d_{\nu}^{\dagger}\right)^{x N}\left(b_{0}^{\dagger}\right)^{(1-2 x) N}|0\rangle
$$

Here $e_{\mu}^{\dagger} \equiv \sum_{i} \phi_{\mu}^{*}(i) e_{i}^{\dagger}$ and $d_{\nu}^{\dagger} \equiv \sum_{i} \phi_{\nu}^{*}(i) d_{i}^{\dagger}$ represent 'bose condensation' [15] of the $B^{+}$and $B^{-}$in two different 'extended states' $\phi_{\mu}(i)$ and $\phi_{\nu}(i)$. As the hopping matrix elements of $B^{+}$and $B^{-}$have opposite sign (equation $4)$, they condense in general in different extended states. The operator $b_{0} \equiv \sum_{i j} \phi_{\mathrm{SC}}(i, j) b_{i j}^{\dagger}$ represents the condensation of valence bond pairs $B^{0}-B^{0}$ in an extended state represented by the pair function $\phi_{\mathrm{SC}}(i, j)$. And $\hat{P}$ is a projection operator that ensures the presence of only one of $B^{0}$ or $B^{+}$or $B^{-}$acceptor states in any boron site in the many body wave function, by obeying the local constraint, $d_{i}^{\dagger} d_{i}+e_{i}^{\dagger} e_{i}+\sum_{\sigma} s_{i \sigma}^{\dagger} s_{i \sigma}=1$.

To understand the above variational wave function (equation 6), we wish to state that in a non random case of simple cubic lattice for example, the holons and doublons respectively condense at wave vectors $(0,0,0)$ and $(\pi, \pi, \pi)$, in view of the different signs of the holon and doublon hopping matrix elements in equation 4. Also note that in the RVB theory, the holon and doublon condensation, an apparent charge 'e' condensation [15] is ac- 
tually a book keeping device 16] for the charge ' $2 \mathrm{e}$ ' condensation of physical electron pairs (valence bond pairs).

As mentioned earlier, in view of the random character of our Hamiltonian, one can only make some existence type of statement of the functions $\phi_{\mu}(i), \phi_{\nu}(i)$ and $\phi_{\mathrm{SC}}(i, j)$ from plausibility arguments. For the same reason it is difficult to get quantitative estimate of the superconducting $T_{c}$, from a formally 'exact' gap equation, even within the slave boson mean field analysis. However, we can get a very rough order of magnitude estimate of the superconducting $T_{c}$ using certain 'typical' values of the impurity band parameters using a standard RVB mean field expression, $k_{B} T_{c} \approx \frac{W}{2} e^{-\frac{W}{J}}$. If we assume an impurity band width $W$ around $0.2 \mathrm{eV}$ and superexchange $\mathrm{J}$ around $0.04 \mathrm{eV}$, we get a $T_{c}$ in the range 1 to $10 \mathrm{~K}$. It is important to remark that what is seen experimentally in diamond:B is likely to be some kind of lower bound for a larger intrinsic superconducting $T_{c}$, as inhomogeneities in the real system are likely to affect $T_{c}$ considerably.

What is the order parameter symmetry ? Superconductivity is in a disordered lattice of boron acceptors. Unlike cuprates, where a $d_{x^{2}-y^{2}}$-wave symmetry is compatible with an underlying square lattice symmetry, higher angular momentum states have no compatibility with the underlying random lattice. Hence, diamond:P is likely to go into an extended s-wave superconducting state, there by also respecting Anderson's theorem on dirty superconductors.

The conducting side of the Anderson-Mott transition, in view of residual unscreened short range coulomb repulsion could create a charge density glass of the spontaneously created $B^{+}$and $B^{-}$carriers as well. This will be a competitor to superconductivity.

If our correlation mechanism is at work, the normal state should be an anomalous one. Disorder should lead to certain soft and localized spinon and chargeon quasi particle states. This is likely to lead to normal state anomalies different from cuprates.

Our current proposals, including mechanism of superconductivity, as it stands is very heuristic and based on limited available experimental results. To make further progress and check the validity and consequences of the present proposal, it is important to perform certain experiments: i) precise measurement of the critical concentration $n_{c}$ of the Anderson-Mott insulator to metal transition ii) map out the superconductivity phase diagram by changing the boron concentration iii) look for anomalous $T^{\alpha}$ type power law signals in specific heat and magnetic susceptibility in the insulating phase, characteristic of the valence bond glass phase and hierarchical singlet coupling, iv) 'spin gap' type of behaviour in the normal state on the conducting side, looking for onset of spinon pairing v) normal state transport anomalies on the conducting side and vi) look for superconductivity in the Mott insulator phase by small amount of compensation, say by nitrogen type donor impurities; this will be an external doping of the boron impurity band rather than self-doping.

Our present proposals raise some questions and suggests applicability to other systems. Why superconductivity is absent in Si:P and related systems? Apart from the fact that the energy scales are low, the impurity state orbital degeneracy is high and spin-orbit coupling is relatively high. Hund coupling and reduced band filling resulting from multiple bands is likely to diminish possibility of singlet superconductivity. It will be also interesting to look for new systems and also find out if electron correlation play any important role in estabilishing superconductivity in known impurity band superconductors 17]

I thank Prof S Maekawa for bringing to my attention reference [1], Prof. S Maekawa and Prof H Fukuyama for discussion, hospitality and IFCAM Visiting Professorship at Sendai. An informative discussion on spectrosopy of boron acceptor states in diamond with Prof A K Ramdas in February 2004 is acknowledged.

[1] E.A. Ekimov et al., Nature (London), 428542 (2004)

[2] The properties and Natural and Synthetic Diamonds, Edited by J.E. Field (Academic, London) 1992; H. Kym et al., Phys. Rev. Lett., 834140 (1999)

[3] P.W. May, Phil. Trans. R. Soc. Lond., A358 473 (2000)

[4] R.N. Bhatt and T.M. Rice, Phys. Rev. B23 1920 (1981); A.M. Finkelstein, Sov. Phys. JETP, 5797 (1985); P.A. Lee and T. V. Ramakrishnan, Rev. Mod. Phys., 57287 (1985); D. Belitz and T.R. Kirkpatrick, Rev. Mod. Phys., 66261 (1991)

[5] P.W. Anderson, Science, 2351196 (1987); G. Baskaran, Z. Zou and P.W. Anderson, Sol. St. Commn., 63973 (1987); G. Baskaran and P.W. Anderson, Phys. Rev., B 37580 (1988).

[6] P.W. Anderson et al., cond-mat/0311467

[7] T. Tshepe, J.F. Prinz and M.J.R. Hoch, Diamond Relat. Mater., 81508 (1999)

[8] for a recent review see, E. Bustarret, E. Gheeraert and K. Watanabe, Physica Status Solidi, A 1999 (2003).

[9] G. Baskaran, Phys. Rev. Lett., 90197007(2003)

[10] F.C. Zhang, Phys. Rev. Lett., 90207002 (2003)

[11] T. Sato et al., Phys. Rev. B 6112970 (2000)

[12] R.N. Bhatt, Phys. Rev., 261082 (1982)

[13] R.N. Bhatt and P.A. Lee, Phys. Rev. Lett., 48344 (1982)

[14] M. Milovanovic, S. Sachdev and R.N. Bhatt, Phys. Rev. Lett., 6382 (1989) 48597 (1982); M.A. Paalanen et al. Phys. Rev. Lett., 61597 (1988); M. Lakner et al., Phys. Rev. 5017064 (1994)

[15] S. A. Kivelson, D. Rokhsar and J. Sethna, Phys. Rev., 388865 (1987)

[16] P.W. Anderson et al., Phys. Rev. Lett., 582790 (1987)

[17] J.K. Hulm et al., in Progress in Low Temperature Physics (ed. C.J. Gorter) Vol. VI, 205-242 (North-Holland, Amsterdam, 1970) 